\title{
CCD PHOTOMETRY OF HIPPARCOS STELLAR SYSTEMS
}

\author{
E. OBLAK AND M. CHARETON \\ Observatoire de Besançon, France ${ }^{\dagger}$
}

With the introduction of CCD detectors, it now appears feasible to obtain accurate photometric data for each of the components of close visual double stars with an angular separations between 1 and 12".

A programme of systematic and homogeneous acquisition of precise colour indices for several thousand components of double and multiple systems has been carried out by a European Network of Laboratories (see footnote) and supported by an ESO key programme.

The principle scientific objective is to provide the missing photometric data needed to supplement the high quality of Hipparcos astrometric data. VRI CCD photometry has been carried out in observatories in both hemispheres (La Palma, Calar Alto, OHP). The relative position of the components is obtain as a by-product thanks to CCD astrometric observations developed by the group in a few numbers of selected fields in right ascension. Since neither the crowded fields, nor the isolated star (aperture) software packages are convenient, specific reduction softwares had to be developed for PSF-fitting of overlapping star profiles.

Both the photometry and astrometry of visual double stars are being obtained at accuracy of 0.01 level in angular separation and photometry. The preliminary comparison based on the results of the observational campaign and the Hipparcos solutions permits the correction of the grid step ambiguity of the satellite and corrects for color term effects giving, after correction, more consistent results from the two methods.

A photometric database of stellar systems is compiled and will be generalized to all categories of stellar systems.

A parallel work consists of examining and correcting the data on stellar systems of the 'Centre de Données astronomiques' of Strasbourg (CDS).

${ }^{\dagger}$ with the collaboration of A.N. Argue, P. Brosche, J. Cuypers, M. Geffert, M. Grenon, J.L. Halbwachs, M. Irwin, G. Jasniewicz, E. Martin, J.L. Mermilliod, F. Mignard, W. Seggewiss, D. Sinachopoulos, E. Van Dessel, S. Zola. 